\title{
Correction to: Development of a column-switching LC-MS/MS method of tramadol and its metabolites in hair and application to a pharmacogenetic study
}

\author{
Hyerim Yu ${ }^{1} \cdot$ Minje Choi $^{1} \cdot$ Jung-Hee Jang $^{2} \cdot$ Byoungduck Park $^{1} \cdot$ Young Ho Seo $^{1} \cdot$ Chul-Ho Jeong $^{1} \cdot$ \\ Jung-Woo Bae $^{1} \cdot$ Sooyeun Lee ${ }^{1}$
}

Published online: 12 January 2019

(c) The Pharmaceutical Society of Korea 2019

\section{Correction to: Arch. Pharm. Res. (2018) 41:554-563 https://doi.org/10.1007/s12272-018-1013-7}

In the original version of the article titled "Development of a column-switching LC-MS/MS method of tramadol and its metabolites in hair and application to a pharmacogenetic study", published in 41(5):554-563 (https://doi.org/10. 1007/s12272-018-1013-7), unfortunately an important statement was missing in the Clinical study section by the author.

The following statement: "The clinical study was approved by the Institutional Review Board of Keimyung University (Deagu, Republic of Korea, approval number: 40525-201509-BR-70-02) and Kyungpook National University Hospital (Daegu, Republic of Korea, approval number of clinical trial: 2016-08-005) and carried out at the Kyungpook National University Hospital Clinical Trial Center (Daegu, Republic of Korea)."

Should read: "Written informed consent was obtained from all subjects. The study was performed in accordance with the guidelines of the Declaration of Helsinki on biomedical research involving human subjects and was approved by the Institutional Review Board of Keimyung University (Deagu, Republic of Korea, approval number: 40525-201509-BR-70-02) and Kyungpook National University Hospital (Daegu, Republic of Korea, approval number of clinical trial: 2016-08-005) and carried out at the Kyungpook National University Hospital Clinical Trial Center (Daegu, Republic of Korea)."
The original article can be found online at https:// doi.org/10.1007/s12272-018-1013-7.

\section{Jung-Woo Bae}

jwbae11@kmu.ac.kr

$\triangle$ Sooyeun Lee sylee21@kmu.ac.kr

1 College of Pharmacy, Keimyung University, 1095 Dalgubeoldaero, Dalseo-gu, Daegu 42601, Republic of Korea

2 School of Medicine, Keimyung University, 1095 Dalgubeoldaero, Dalseo-gu, Daegu 42601, Republic of Korea 